\title{
latrogenic Ligation of the Left Subclavian Artery During Palliative Left Pneumonectomy
}

\author{
Mark Tatterton $^{\mathrm{a}, \mathrm{b}}$, David Russell ${ }^{\mathrm{a}}$
}

\begin{abstract}
Iatrogenic injury to the subclavian artery during thoracic surgery is rare. Here we describe a rare case of woman who developed an ischemic left upper limb as a complication of a palliative left pneumenectomy, together with her subsequent management.
\end{abstract}

Keywords: Subclavian artery; Ligation; Trauma; Bypass

\section{Introduction}

Iatrogenic injury to the subclavian artery is a well described complication of attempted central venous access [1, 2]. Although an obvious vessel at risk due to its vicinity with thoracic structures, iatrogenic injury to the subclavian artery during thoracic surgery is much less well described.

\section{Case Report}

A 42-year-old lady underwent a difficult palliative left pneumenectomy for a T3, N1, M0, G2 squamous cell carcinoma of the left lung. Surgery was complicated by the fact that the patient had a large cavitating abscess involving most of the left hemithorax associated with the tumor. A very experienced thoracic surgeon performed the case and noted several times during the surgery the difficulty of the dissection due to adhesive tissue. During apical dissection, the left internal mammary artery was torn from the subclavian artery which led to significant intra-operative hemorrhage of over

\footnotetext{
Manuscript accepted for publication October 5, 2013

${ }^{a}$ Leeds Vascular Institute, Leeds General Infirmary, Great George Street, Leeds, LS1 3EX, UK

${ }^{\mathrm{b}}$ Correspondance author: Mark Tatterton, Leeds Vascular Institute, Leeds General Infirmary, Great George Street, Leeds, LS1 3EX, UK. Email: mtatterton@doctors.org.uk
}

doi: http://dx.doi.org/10.4021/jmc1539w

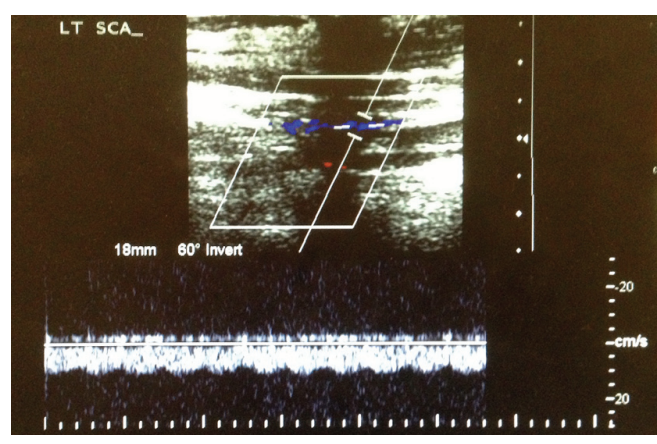

Figure 1. Pre-operative arterial duplex demonstrating low velocity flow in the left subclavian artery.

$1 \mathrm{~L}$. In order to gain rapid control, a surgical clip ligator was used successfully to control hemorrhage. The rest of the procedure went uneventful.

The patient went from recovery directly to the ward. Whilst on the ward, the patient reported pain near the left clavicle and throughout the left arm, parasthesia and reduced power in the hand and forearm. The clinical team examined the patient and found a cold arm with no peripheral pulses. The vascular team was quickly contacted and on review, it was clear the patient had an ischemic arm with evolving neurology. An urgent bedside arterial duplex was performed by a vascular radiologist. Due to surgical emphysema, the

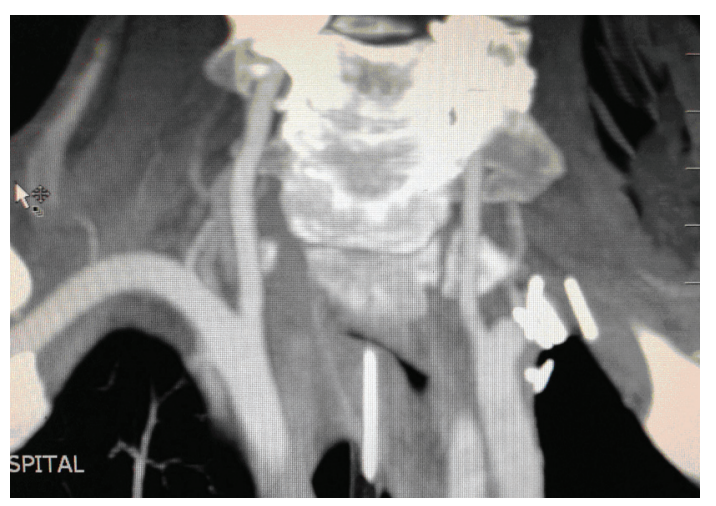

Figure 2. CT angiogram demonstrating surgical clips and complete occlusion of left subclavian artery. 


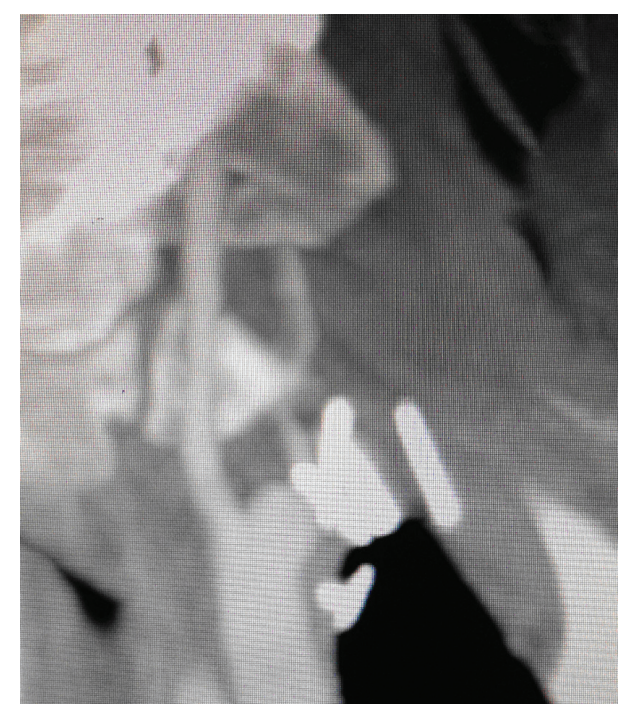

Figure 3. Enlarged version of CT angiogram demonstrating occluded left subclavian artery.

proximal subclavian artery was not demonstrated however the distal subclavian artery demonstrated very low velocity ischemic flow, continuing into the brachial and radial arteries (Fig. 1). An urgent CT angiogram was performed which confirmed complete occlusion of the first part of the left subclavian artery immediately after the origin of the left thyrocervical trunk due to surgical clips (Fig. 2, 3).

In view of the fact that the arm was acutely ischemic with neurology, an urgent left carotid to distal subclavian bypass was perfomed using right-sided cephalic vein. Post operatively the patient suffered no immediate complications. An arterial duplex scan performed five days post surgery demonstrated excellent tri-phasic flow into the distal subclavian and axillary arteries (Fig. 4).

\section{Discussion}

Iatrogenic ligation of the subclavian artery is a very rare com-



Figure 4. Postoperative arterial duplex demonstrating triphasic flow through the left subclavian artery post bypass.

plication of pneumonectomy. As with all significant arterial bleeding, the avoidance of placing clips blind is paramount to prevent surrounding tissue injury. If bleeding cannot be controlled safely, urgent assistance from a vascular surgeon/ interventional radiologist should be sought.

\section{Disclosure}

No conflict of interests. No grant support.

\section{References}

1. Cayne NS, Berland TL, Rockman CB, Maldonado TS, Adelman MA, Jacobowitz GR, Lamparello PJ, et al. Experience and technique for the endovascular management of iatrogenic subclavian artery injury. Ann Vasc Surg. 2010;24(1):44-47.

2. Guilbert MC, Elkouri S, Bracco D, Corriveau MM, Beaudoin N, Dubois MJ, Bruneau L, et al. Arterial trauma during central venous catheter insertion: Case series, review and proposed algorithm. J Vasc Surg. 2008;48(4):918-925; discussion 925. 